\title{
The Dirichlet Boundary Conditions and Related Natural Boundary Conditions in Strengthened Sobolev Spaces for Discretized Parabolic Problems
}

\author{
E. D’YAKONOV \\ Department of Computer Mathematics and Cybernetics, Moscow State University, Moscow, 119899, Russia
}

(Received 21 April 1999)

\begin{abstract}
Correctness of initial boundary value problems and their discretizations are analyzed under unusual second-order boundary conditions, which can be considered as natural boundary conditions in strengthened Sobolev spaces and as improvements (in some cases) of the classical Dirichlet boundary conditions. Special attention is paid to optimal perturbation estimates for new variants of the penalty method with respect to the Dirichlet conditions.
\end{abstract}

Keywords: The Dirichlet and related natural boundary conditions, Strengthened Sobolev spaces, Parabolic problems

\section{INTRODUCTION}

The Dirichlet boundary conditions are of fundamental importance in mathematical physics and other fields of science. They serve as a means to isolate the problem under consideration in a given domain $\Omega \subset \mathbf{R}^{d}$ from the outside world; in what follows, we assume that $\Omega$ is a bounded domain with Lipschitz piecewise smooth boundary $\Gamma$.

Other boundary conditions are possible. Sometimes they lead to a better description of what happens on the boundary; in the case, for example, of an elliptic variational problem in the Sobolev space $H_{0}^{1}(\Omega) \equiv \stackrel{\circ}{W}_{2}^{1}(\Omega)$, there is a common opinion that the homogeneous Dirichlet conditions can be treated in terms of the penalty method as a limit of natural boundary conditions of the type $\left(\partial u_{\varepsilon} / \partial \vec{n}\right)+(1+1 / \varepsilon) u_{\varepsilon}=0$, where $\vec{n}$ is the unit vector of the outer normal to the boundary, $\varepsilon \rightarrow+0$ (see Courant, 1943; Babushka, 1973; 1987; Sobolevskii, 1981; Bramble, 1981; Glowinski, 1983). These conditions are connected with the additional term (penalty term) $F(u) \equiv(1+1 / \varepsilon)|u|_{0, \Gamma}^{2}\left(|u|_{0, \Gamma}^{2} \equiv\right.$ $\left.\|u\|_{L_{2}(\Gamma)}^{2}\right)$ in the minimized energy functional. Sometimes conditions of such a type have a good physical sense and improve the original Dirichlet conditions - this is the case, for example, in theory of elasticity when springs on the boundary are allowed; sometimes the new conditions are considered as very artificial (see, for example, Glowinski (1983), where they were applied to problems of hydrodynamics). From the mathematical point of view, the penalty 
term might be also considered as rather weak because of additional smoothness requirements on the solutions for obtaining estimates of the type

$$
\left\|u_{\varepsilon}-u\right\|_{W_{2}^{1}(\Omega)}+\left|u_{\varepsilon}-u\right|_{0, \Gamma}=O(\varepsilon) .
$$

It was shown recently (see D'yakonov, 1997a,b) that such and even stronger estimates can be proved assuming only correctness of the original problem if apply the penalty term $\bar{F}(u) \equiv(1+1 / \varepsilon)\|u\|_{W_{2}^{\nu}(\Gamma)}^{2}$ with $\nu \geq 1 / 2$ and treat the arising problem in the corresponding strengthened Sobolev space. The case $\nu=1$ is the most remarkable since it even allows some domains with slits and has good perspectives from the computational point of view; moreover, it often has an obvious physical sense. For example, in theory of elastic membranes, it corresponds to the presence of string (stiffener) on the boundary; similar problems for plates and shells with stiffeners are of special importance in many applications (see Courant, 1943; D'yakonov, 1996a; Ciarlet, 1997 and references therein); it should be noted that S.P. Timoshenko was the first to set them (in a pre-Hilbert space) in 1915; certain problems of hydrodynamics with surface tension can be also set in similar strengthened Sobolev spaces (see D'yakonov, 1997c); importance of relevant secondorder boundary conditions was also underlined in the study of problems on unbounded domains (see Duong and Joly, 1994; Sheen, 1993).

The goal of the present paper is a generalization of the approach indicated above to the case of parabolic problems. Correctness of the initial boundary value problems (with an emphasis on their discretizations with respect to the time) is analyzed by the energy method under special choice of energy spaces associated with the strengthened Sobolev spaces.

Special attention is paid to optimal perturbation estimates for the variant of the penalty method mentioned above (with respect to the the homogeneous Dirichlet conditions). The results obtained also yield understanding of the mechanism of splitting of the parabolic problem into separate ones in subdomains with the homogeneous Dirichlet conditions on the boundaries of these subdomains.

In this paper, only real Hilbert spaces and bounded operators are used; the normed linear space of linear bounded operators mapping $U$ into $F$ is denoted by $\mathcal{L}(U ; F) ; \quad\|L\| \equiv\|L\|_{U \mapsto F} \equiv$ $\sup _{v \neq 0}\|L v\|_{F}\|v\|_{U}^{-1} ; \quad \mathcal{L}(H) \equiv \mathcal{L}(H ; H) ; \quad \operatorname{Ker} L \equiv$ $\{v: L v=0\} \equiv$ the kernel (null-space) of the operator $L ; \operatorname{Im} L \equiv L\{U\} \equiv$ the image (range) of the operator $L ; I \equiv$ the identity operator; $H^{*} \equiv$ the linear space of bounded linear functionals $l$ mapping $H$ into $\mathbf{R} ; A^{*} \equiv$ the adjoint operator to $A \in \mathcal{L}\left(H_{1} ; H_{2}\right)$ $\left((A u, v)_{H_{2}}=\left(u, A^{*} v\right)_{H_{1}}, \forall u \in H_{1}, v \in H_{2}\right) ; A_{s} \equiv$ the symmetric part of $A \in \mathcal{L}(H)$, i.e., $A_{s} \equiv 2^{-1}\left(A+A^{*}\right)$; $\mathcal{L}^{+}(H)$ denotes the set of linear, symmetric, and positive definite operators in $\mathcal{L}(H) ; H(B) \equiv$ the Hilbert space differing from $H$ only by inner product defined by $B \in \mathcal{L}^{+}(H)$, namely $(u, v)_{H(B)} \equiv$ $(u, v)_{B} \equiv(B u, v)_{H} \equiv(B u, v)$.

For nonnegative functions $f(h)$ and $g(h), f(h) \asymp$ $g(h)$ implies that there exist positive constants $\kappa_{0}$ and $\kappa_{1}$ such that $\kappa_{0} g(h) \leq f(h) \leq \kappa_{1} g(h)$; we also make use of this notation for norms, quadratic functionals and operators, e.g., $L \asymp I \Leftrightarrow L \in$ $\mathcal{L}^{+}(H)$. For simplicity, we consider only $\Omega \subset \mathbf{R}^{2}$; the space variables are denoted by $x_{1}$ and $x_{2}$ with $x \equiv\left[x_{1}, x_{2}\right] ;|u|_{0, \Omega}^{2} \equiv\|u\|_{L_{2}(\Omega)}^{2}$; the time variable is $t \equiv x_{0} \in[0, T] ; \quad \bar{x} \equiv\left[x_{0}, x_{1}, x_{2}\right] \in Q_{T} \equiv \Omega \times[0, T] ;$ $D_{s} u \equiv \partial u / \partial x_{s}, s=0,1,2 ;|\nabla u| \equiv\left[\left(D_{1} u\right)^{2}+\left(D_{2} u\right)^{2}\right]^{1 / 2}$; $(u, v)_{1, \Omega} \equiv\left(D_{1} u, D_{1} v\right)_{0, \Omega}+\left(D_{2} u, D_{2} v\right)_{0, \Omega} ; \quad|u|_{1, \Omega} \equiv$ $\left(|\nabla u|^{2}, 1\right)_{0, \Omega}^{1 / 2}$;

$$
\|u\|_{1, \Omega}^{2} \equiv|u|_{0, \Omega}^{2}+|u|_{1, \Omega}^{2} \equiv\|u\|_{H_{1}(\Omega)}^{2} .
$$

\subsection{Variational Problems with Linear Constraints and Corresponding Problems with Strongly Saddle Operators}

We briefly recall the most important facts related to variational problems with linear constraints (see, Brezzi, 1974; D'yakonov, 1996a and references therein) in a given Hilbert space $H_{1}$. The variational 
problem is to find

$$
u_{1}=\arg \min _{v_{1} \in V_{1}}\left[I_{2}\left(v_{1}\right)-2 l\left(v_{1}\right)\right],
$$

with a quadratic functional $I_{2}\left(v_{1}\right) \asymp\left\|v_{1}\right\|_{H_{1}}^{2}$ and linear functional $l \in H_{1}^{*}$; the space $V_{1}$ of admissible functions consists of functions $v_{1}$ such that $L_{2,1} v_{1}=$ 0 for a given $L_{2,1} \in \mathcal{L}\left(H_{1} ; H_{2}\right)$ that is $V_{1}=\operatorname{Ker} L_{2,1}$.

The standard penalty method for $(0.1)$ consists in setting a sequence of unconstrained problems

$$
u_{1, \varepsilon}=\arg \min _{v_{1} \in H_{1}}\left[I_{2}\left(v_{1}\right)+1 / \varepsilon\left\|L_{2,1} u_{1}\right\|_{H_{2}}^{2}-2 l\left(v_{1}\right)\right],
$$

with the penalty parameter $\varepsilon \rightarrow+0$.

The classical Lagrange approach (the Lagrange multiplier method) to the problem (0.1) is to replace it by the problem

$$
L u \equiv\left[\begin{array}{cc}
L_{1,1} & L_{1,2} \\
L_{2,1} & 0
\end{array}\right]\left[\begin{array}{l}
u_{1} \\
u_{2}
\end{array}\right]=\left[\begin{array}{c}
f_{1} \\
0
\end{array}\right],
$$

a Hilbert space $H=H_{1} \times H_{2}$, where the additional function $u_{2}$ plays the role of the Lagrangian multiplier,

$$
L_{1,1} \asymp I_{1}, \quad L_{1,2}=L_{2,1}^{*} .
$$

The modern formulation of problems (0.3), (0.4) (saddle-point problems arising from Lagrangian multipliers) and more general ones of type

$$
L_{\varepsilon} u_{\varepsilon} \equiv\left[\begin{array}{cc}
L_{1,1} & L_{1,2} \\
L_{2,1} & -\varepsilon I_{2}
\end{array}\right]\left[\begin{array}{l}
u_{1, \varepsilon} \\
u_{2, \varepsilon}
\end{array}\right]=\left[\begin{array}{c}
f_{1} \\
0
\end{array}\right]
$$

(see (0.2)) is based on the use of special types of operators $L_{2,1}$ which can be described as normally invertible operators (operators with $\operatorname{Im} L_{2,1}=H_{2}$ ); equations with them are called everywhere solvable (see Krein, 1971). They correspond to a particular and very well known case of normally solvable operators (see Krein, 1971; Rempel and Schulze, 1985; Trenogin, 1980; D'yakonov, 1996a and references therein) which are defined as operators with
$\operatorname{Im} L_{2,1}$ being subspaces in $H_{2}$ (operators with closed images); if $L_{2,1}$ is a normally solvable operator, then $H_{1}$ is an orthogonal sum of $\operatorname{Ker} L_{2,1}$ and $\operatorname{Im} L_{2,1}^{*}$, i.e.,

$$
H_{1}=\operatorname{Ker} L_{2,1} \oplus \operatorname{Im} L_{1,2} .
$$

A normally invertible operator $L_{2,1}$ yields a oneto-one mapping of the Hilbert space $\operatorname{Im} L_{1,2}$ (orthogonal complement in $H_{1}$ to $\operatorname{Ker} L_{2,1}$ ) into $H_{2}$ (see (0.6)) and by the Banach theorem this mapping is invertible and the corresponding inverse $L_{2,1}^{(-1)} \equiv L_{2,1}^{\dagger}$ is such that

$$
\left\|L_{2,1}^{\dagger}\right\| \equiv \sigma^{-1}<\infty
$$

We note that the well-known inf-sup condition,

$$
\inf _{u_{2} \in H_{2}} \sup _{u_{1} \in H_{1}} \frac{\left(L_{2,1} u_{1}, u_{2}\right)_{H_{2}}}{\left\|u_{1}\right\|_{H_{1}}\left\|u_{2}\right\|_{H_{2}}} \geq \sigma>0
$$

is often used instead of $(0.7) ;(0.8)$ can be written in the form

$$
\left\|L_{2,1}^{*} u_{2}\right\|_{H_{1}} \geq \sigma\left\|u_{2}\right\|_{H_{2}}, \quad \forall u_{2} \in H_{2}
$$

(see D'yakonov, 1996a; Girault and Raviart, 1986; Pironneeau, 1989 and references therein) which implies (0.6). It is also worth noting that $(0.7)$ yields inclusions

$$
\begin{aligned}
\operatorname{sp} L_{2,1} L_{2,1}^{*} & \subset\left[\sigma^{2},\left\|L_{2,1}\right\|^{2}\right], \\
\operatorname{sp} L_{2,1}^{*} L_{2,1} \backslash 0 & \subset\left[\sigma^{2},\left\|L_{2,1}\right\|^{2}\right],
\end{aligned}
$$

where $\operatorname{sp} A$ denotes the spectrum of $A$ (see D'yakonov, 1986); for $L_{2,1}$ associated with the divergence operators, first results (in pre-Hilbert spaces) about such spectrums were obtained in Cosserat (1898).

The operator $L_{\varepsilon}$ in (0.5) with $\varepsilon \geq 0$ and a normally invertible operator $L_{2,1}$ is called a strongly saddle operator. For such operators, it was proved (see D'yakonov, 1983; 1996a) that $L_{\varepsilon}$ is invertible and

$$
\left\|L_{\varepsilon}^{-1}\right\| \leq K,
$$


where the constant $K$ can be chosen uniformly for all $\varepsilon>0$. This implies that problem (0.5) (or $(0.3)$ ) is correctly posed and the first component of its solution coincides with the solution of problem (0.2) (or (0.1)). Moreover (0.10) implies, for all parameters indicated, that

$$
\left\|u_{\varepsilon}-u_{\varepsilon^{\prime}}\right\|_{H} \leq K_{1}\left|\varepsilon-\varepsilon^{\prime}\right|, \quad \varepsilon \in\left[0, \varepsilon_{0}\right], \varepsilon^{\prime} \in\left[0, \varepsilon_{0}\right],
$$

for a given $\varepsilon_{0}>0$.

Thanks to an understanding of the role of $(0.8)$ and its grid analogs in the theory of progective-grid (finite element) methods and iterative processes, it now seems reasonable to regard problems $(0.5)$ as basic and, instead of problems (0.2) and more general ones involving a large parameter $1 / \varepsilon$, to work with corresponding problems $(0.5)$ (see, e.g., D'yakonov, 1996a).

The results indicated hold for more general problems of type

$$
L_{\varepsilon} u_{\varepsilon} \equiv\left[\begin{array}{cc}
L_{1,1} & L_{1,2} \\
L_{2,1} & -\varepsilon L_{2,2}
\end{array}\right]\left[\begin{array}{l}
u_{1, \varepsilon} \\
u_{2, \varepsilon}
\end{array}\right]=\left[\begin{array}{l}
f_{1} \\
f_{2}
\end{array}\right]
$$

with $L_{2,2} \geq 0$. Moreover, the corresponding correctness and perturbation theorems (see (0.10) and (0.11)) can be obtained for problems of type (0.12) involving nonsymmetric operators $L_{1,1}$ and $L_{2,2}$ with their symmetric parts $L_{1,1, s}$ and $L_{2,2, s}$, respectively. It suffices to assume that $L_{1,1, s} \in \mathcal{L}^{+}\left(H_{1}\right)$ and $L_{2,2, s} \geq 0$ (see D'yakonov, 1996a).

\subsection{Strengthened Sobolev Spaces and Perturbation of the Dirichlet Conditions}

The most significant feature of the problems we study here is that they involve setting in strengthened Sobolev spaces $G_{1, m}(\Omega ; \Gamma) \equiv G_{1, m}(\Gamma \equiv \partial \Omega, m>$ $1 / 2)$ instead of the classical Sobolev space $W_{2}^{1}(\Omega) \equiv$ $H^{1}(\Omega)$ (see D'yakonov, 1996a; 1997a,b,c,d; Ciarlet, 1997 and references therein). In the case of smooth $\Gamma$ we define $G_{1, m}$ as a subset of functions in $H^{1}(\Omega)$ such that their traces on $\Gamma$ belong to $W_{2}^{m}(\Gamma)$, so we may define the norm by

$$
\|v\|_{G_{1, m}}^{2} \equiv\|v\|_{1, \Omega}^{2}+\left\|\operatorname{Tr}_{\Gamma} v\right\|_{W_{2}^{m}(\Gamma)}^{2}
$$

(similar spaces defined in terms of Fourier transformations of the corresponding extended functions were considered in Vishik, 1970). We emphasize that the trace operator $\operatorname{Tr} \equiv \operatorname{Tr}_{\Gamma}$ is understood in the standard way as an element of $\mathcal{L}\left(H^{1}(\Omega) ; L_{2}(\Gamma)\right)$. If $\Gamma$ is not smooth but consists of several smooth $\operatorname{arcs} \Gamma_{i}$ then (0.13) should be replaced by

$$
\|v\|_{G_{1, m}}^{2} \equiv\|v\|_{1, \Omega}^{2}+\sum_{i}\left\|\operatorname{Tr}_{\Gamma_{i}} v\right\|_{W_{2}^{m}\left(\Gamma_{i}\right)}^{2}
$$

This space $G_{1, m}$ is a Hilbert space and traces of its elements on $\Gamma$ can be considered as continuous functions (almost everywhere, see D'yakonov, 1997c,d). The most important case of (0.13) and (0.14) is connected with $m=1$ when

$$
\begin{aligned}
\|v\|_{G_{1,1}}^{2} & \equiv\|v\|_{1, \Omega}^{2}+\|v\|_{1, \Gamma}^{2}, \\
\|v\|_{1, \Gamma}^{2} & \equiv \sum_{i}\left\|\operatorname{Tr}_{\Gamma_{i}} v\right\|_{H^{1}\left(\Gamma_{i}\right)}^{2} ;
\end{aligned}
$$

this Hilbert space of traces will be denoted by $G_{2}(\Gamma) \equiv G_{2}$ and we prefer to write $G_{1}$ instead of $G_{1,1}$. The fundamental for the result of our analysis is connected with consideration of restriction of the operator $\operatorname{Tr} \in \mathcal{L}\left(H^{1}(\Omega) ; L_{2}(\Gamma)\right)$ to our space $G_{1}$ (we denote it by the same symbol); this restriction (see D'yakonov, 1997b) is such that

$$
\operatorname{Tr} \in \mathcal{L}\left(G_{1} ; G_{2}\right), \quad \operatorname{Im} \operatorname{Tr}=G_{2}
$$

To specify applications of the approach indicated to the homogeneous Dirichlet conditions, we start by indicating that $H_{0}^{1}(\Omega) \equiv \stackrel{\circ}{W}_{2}^{1}(\Omega)$ can be considered as a subspace $V_{1}$ of our strengthened Sobolev space $G_{1,1}(\Omega ; \Gamma) \equiv G_{1} \subset H^{1}(\Omega)$ (see $(0.15)-(0.17)$ ). Hence, the original variational problem

$$
u_{1}=\arg \min _{v_{1} \in H_{0}^{1}(\Omega)}\left[I_{2}^{0}\left(v_{1}\right)-2 l\left(v_{1}\right)\right],
$$


with

$$
I_{2}^{0}\left(v_{1}\right) \asymp\left\|v_{1}\right\|_{1, \Omega}^{2}, \quad v_{1} \in H^{1}(\Omega)
$$

can be easily reformulated as problem (0.1) in the space $V_{1} \subset G_{1}$ with

$$
I_{2}\left(v_{1}\right) \equiv I_{2}^{0}\left(v_{1}\right)+\left\|v_{1}\right\|_{1, \Gamma}^{2} \asymp\left\|v_{1}\right\|_{G_{1}}^{2}, \quad \forall v_{1} \in G_{1} ;
$$

or even

$$
I_{2}\left(v_{1}\right) \equiv I_{2}^{0}\left(v_{1}\right)+\left|v_{1}\right|_{1, \Gamma}^{2} \asymp\left\|v_{1}\right\|_{G_{1}}^{2} .
$$

The second example is connnected with the original problem (see (0.18)) under a more general than (0.19) condition of type

$$
I_{2}^{0}\left(v_{1}\right) \asymp\left\|v_{1}\right\|_{1, \Omega}^{2}, \quad v_{1} \in H_{0}^{1}(\Omega) .
$$

Then (0.20) holds.

Finally, under $(0.22)$ it is possible to take a subspace $G_{1}^{\prime} \subset G_{1}$ in the role of $H_{1}$ in (0.1) and deal with (0.21). This is the case if elements of $G_{1}^{\prime}$ are such that $\left(v_{1}, 1\right)_{0, \Gamma}=0$ (see D'yakonov, 1997a,b).

\section{DISCRETIZED IN TIME PARABOLIC PROBLEMS}

\subsection{Original Basic Parabolic Problems in Strengthened Sobolev Spaces}

For consideration of nonstationary problems, it is convenient to rewrite the related stationary problems (0.2) and (0.5) using bilinear forms $b_{r, l}$, defined and bounded on $H_{l} \times H_{r}$ and connected with the operators $L_{r, l}$ by the standard equalities $b_{r, l}\left(v_{l} ; v_{r}\right)=\left(L_{r, l} v_{l}, v_{r}\right), r \in[1,2], l \in[1,2]$. Then $(0.5)$ in case of $(0.1),(0.18)-(0.20)$ is just the problem of finding $u \in H$ such that

$$
\left.\begin{array}{rlrl}
b_{1,1}\left(u_{1} ; v_{1}\right)+b_{1,2}\left(u_{2} ; v_{1}\right) & =l_{1}\left(v_{1}\right), & & \forall v_{1} \in H_{1}, \\
b_{2,1}\left(u_{1} ; v_{2}\right)-\varepsilon b_{2,2}\left(u_{2} ; v_{2}\right) & =0, & & \forall v_{2} \in H_{2},
\end{array}\right\}
$$

where $H_{1} \equiv G_{1,1}(\Omega ; \Gamma), H_{2} \equiv H^{1}(\Gamma), b_{1,1}\left(u_{1} ; v_{1}\right)$ is symmetric,

$$
\begin{gathered}
b_{1,1}\left(v_{1} ; v_{1}\right) \equiv \bar{I}_{2}\left(v_{1}\right) \asymp\left\|v_{1}\right\|_{H_{1}}^{2}, \\
b_{2,2}\left(u_{2} ; v_{2}\right)=\left(u_{2}, v_{2}\right)_{H_{2}}, \\
b_{1,2}\left(u_{2} ; v_{1}\right) \equiv b_{2,1}\left(v_{1} ; u_{2}\right) \equiv\left(\operatorname{Tr} v_{1}, u_{2}\right)_{1, \Gamma}, \\
\forall v_{1} \in H_{1}, \forall u_{2} \in H_{2} .
\end{gathered}
$$

Now, on the basis of (1.1)-(1.3) and as a typical example of nonstationary problems, we consider a sequence of stationary problems

$$
\left.\begin{array}{c}
\left(\left[u_{1}^{n+1}-u_{1}^{n}\right] / \tau ; v_{1}^{n+1}\right)_{0, \Omega}+b_{1,1}\left(u_{1}^{n+1} ; v_{1}^{n+1}\right) \\
\quad+b_{1,2}\left(u_{2}^{n+1} ; v_{1}^{n+1}\right)=l_{1}^{n+1}\left(v_{1}^{n+1}\right), \\
b_{2,1}\left(u_{1}^{n+1} ; v_{2}^{n+1}\right)-\varepsilon b_{2,2}\left(u_{2}^{n+1} ; v_{2}^{n+1}\right)=0,
\end{array}\right\}
$$

where $\tau \equiv T / n^{*}, l^{n+1} \in H_{1}^{*}, v_{r}^{n+1}$ refers to arbitrary elements of $H_{r}, r=1,2, n=0, \ldots, n^{*}-1$.

It is well known that if we take here $H_{1}=$ $H_{0}^{1}(\Omega) \equiv \stackrel{\circ}{W}_{2}^{1}(\Omega)$ and $\varepsilon=0$, then (1.4) corresponds to the well-known implicit semidiscretization with respect to $t$ of a parabolic equation $D_{0} u+L^{0} u=f$ with the homogeneous Dirichlet conditions on the lateral surface of the cylinder $Q_{T} \equiv \bar{\Omega} \times[0, T]$ (spatial variables remain continuous). Thus (1.4) with $H_{1} \equiv G_{1,1}(\Omega ; \Gamma)$ can be treated as a perturbation of the nonstationary problem with the homogeneous Dirichlet conditions. We concentrate on these problems to attain the desired similarity with the stationary ones and avoid introduction of new spaces that arise in dealing with continuous $t$ and correspond to special strengthening of the Sobolev space $H^{1}\left(Q_{t}\right)$.

\subsection{A Priori Estimates and Correctness}

Hereafter, $H_{1}$ and $H_{2}$ are Hilbert spaces, and the original problem (see (1.4) with fixed $n$ ) is formulated in the Hilbert space $H \equiv H_{1} \times H_{2}$ as the 
operator equation

$$
\begin{aligned}
& {\left[\begin{array}{c}
M_{0}\left[u_{1}^{n+1}-u_{1}^{n}\right] / \tau+L_{1,1} u_{1}^{n+1}+L_{1,2} u_{2}^{n+1} \\
L_{2,1} u_{1}^{n+1}-\varepsilon u_{2}^{n+1}
\end{array}\right]} \\
& \quad=\left[\begin{array}{c}
f_{1}^{n+1} \\
0
\end{array}\right]
\end{aligned}
$$

with

$$
\begin{aligned}
& \left(M_{0} u_{1}, v_{1}\right)_{H_{1}} \equiv\left(u_{1}, v_{1}\right)_{0, \Omega},\left\|u_{1}\right\|_{M_{0}} \equiv\left|u_{1}\right|_{0, \Omega}, \\
& \forall u_{1} \in H_{1}, \forall v_{1} \in H_{1},
\end{aligned}
$$

$\left(f^{n+1}, v_{1}\right)_{H_{1}} \equiv l^{n+1}\left(v_{1}\right), \forall v_{1} \in H_{1}$, and $u_{1}^{0}=0$ (this can be assumed without loss of generality). Note that $M_{0} \in \mathcal{L}\left(H_{1} ; H_{1}\right)$ and $M=M^{*}>0$; moreover,

$$
\left\|M_{0} u_{1}\right\|_{H_{1}} \leq \gamma\left|u_{1}\right|_{0, \Omega}, \quad \forall u_{1} \in H_{1} ; \gamma \equiv\left\|M_{0}\right\|
$$

THEOREM 1.1 Let $H_{1} \equiv G_{1,1}(\Omega ; \Gamma), H_{2} \equiv H^{1}(\Gamma)$, and operator $L$ in (0.3) with $\varepsilon \geq 0$ be a strongly saddle operator in the space $H \equiv H_{1} \times H_{2}$. Then

$$
L_{\varepsilon} \equiv\left[\begin{array}{cc}
1 / \tau M_{0}+L_{1,1} & L_{1,2} \\
L_{2,1} & -\varepsilon I_{2}
\end{array}\right]
$$

with $M_{0}$ from (1.6) is also a strongly saddle operator in the space $H$.

Proof Since $M_{0} \in \mathcal{L}\left(H_{1} ; H_{1}\right)$ and $M=M^{*}>0$, we conclude that $\left[1 / \tau M_{0}+L_{1,1}\right] \asymp I_{1}$. Therefore, $L_{\varepsilon}$ from (1.7) has the desired properties.

Theorem 1.1 implies that problem (1.5) and even more general problem

$$
\left[\begin{array}{c}
\bar{\partial}_{0} M_{0} u_{1}^{n}+L_{1,1} u_{1}^{n}+L_{1,2} u_{2}^{n} \\
L_{2,1} u_{1}^{n}-\varepsilon u_{2}^{n}
\end{array}\right]=\left[\begin{array}{l}
f_{1}^{n} \\
f_{2}^{n}
\end{array}\right],
$$

with $f_{2}^{n} \in H_{2}, n=1, \ldots, k \leq n^{*} \equiv T / \tau$, has a unique solution (here and elsewhere, we prefer to write $\partial_{0} u_{1}^{n}$ instead of $\left[u_{1}^{n}-u_{1}^{n-1}\right] / \tau$.

Note also that problem (1.5) with $\varepsilon>0$ is reduced to

$$
M_{0} \bar{\partial}_{0} u_{1}^{n+1}+L_{1,1} u_{1}^{n+1}+\frac{1}{\varepsilon} L_{1,2} L_{2,1} u_{1}^{n+1}=f_{1}^{n+1}
$$

this type of problem is often used in the study of the Stokes problems (see D'yakonov, 1996a; Kobelkov, 1994; Sobolevskii and Vasil'ev, 1978).

THEOREM 1.2 Let the conditions of Theorem 1.1 be satisfied. Then, for the solution of (1.5), the a priori estimate

$$
\begin{aligned}
& \left\|u_{1}\left(t_{k}\right)\right\|_{M_{0}}^{2}+\tau \sum_{n=1}^{k}\left\|u_{1}^{n}\right\|_{H_{1}}^{2}+\varepsilon \tau \sum_{n=1}^{k}\left\|u_{2}^{n}\right\|_{H_{2}}^{2} \\
& \leq K \tau \sum_{n=1}^{k}\left\|f_{1}^{n}\right\|_{H_{1}}^{2}
\end{aligned}
$$

holds, where $t_{k} \equiv k \tau \leq T$ and $K$ is independent of $k \leq n^{*}$ and $\varepsilon \geq 0$.

Proof By (1.5), we have

$$
\begin{gathered}
\tau \sum_{n=0}^{k-1}\left(\left(M_{0} \bar{\partial}_{0} u_{1}^{n+1}, u_{1}^{n+1}\right)_{H_{1}}+\left(L_{1,1} u_{1}^{n+1}, u_{1}^{n+1}\right)_{H_{1}}\right. \\
\left.\quad+\varepsilon\left(u_{2}^{n+1}, u_{2}^{n+1}\right)_{H_{2}}\right)=\tau \sum_{n=0}^{k-1}\left(f_{1}^{n+1}, u_{1}^{n+1}\right)_{H_{1}} .
\end{gathered}
$$

For the first term on the left-hand side of (1.11), we have

$$
\begin{aligned}
& \tau \sum_{n=0}^{k-1}\left(M_{0} \bar{\partial}_{0} u_{1}^{n+1}, u_{1}^{n+1}\right)_{H_{1}} \\
& \quad=\sum_{n=0}^{k-1}\left(\left(u_{1}^{n+1}-u_{1}^{n}\right), u_{1}^{n+1}\right)_{M_{0}} \geq \frac{1}{2}\left\|u_{1}^{k}\right\|_{M_{0}}^{2}
\end{aligned}
$$

(recall that $u_{1}^{0}=0$ ). Therefore, the left-hand side of (1.11) is easily estimated from below as

$$
\delta \tau \sum_{n=1}^{k}\left\|u_{1}^{n}\right\|_{H_{1}}^{2}+\frac{1}{2}\left\|u_{1}^{k}\right\|_{M_{0}}^{2}+\varepsilon \tau \sum_{n=1}^{k}\left\|u_{2}^{n}\right\|_{H_{2}}^{2}
$$

where $L_{1,1} \geq \delta I_{1}, \quad \delta>0$. The right-hand side of (1.11) is estimated from above as

$$
\begin{aligned}
& \tau \sum_{n=0}^{k-1}\left(f_{1}^{n+1}, u_{1}^{n+1}\right)_{H_{1}} \\
& \quad \leq \frac{\tau \delta}{2} \sum_{n=0}^{k-1}\left\|u_{1}^{n+1}\right\|_{H_{1}}^{2}+\frac{\tau}{2 \delta} \sum_{n=0}^{k-1}\left\|f_{1}^{n+1}\right\|_{H_{1}}^{2}
\end{aligned}
$$


(recall the evident inequality $(f, u) \leq \delta / 2\|u\|^{2}+$ $\left.1 /(2 \delta)\|f\|^{2}, \forall \delta>0\right)$. These two estimates obtained (in combination with (1.11)) lead directly to (1.10).

THEOREM 1.3 Let the conditions of Theorem 1.1 be satisfied and suppose that

$$
\left(f_{1}^{n}, u_{1}\right)_{H_{1}}=\left(g_{1}^{n}, u_{1}\right)_{M_{0}}, \quad \forall u_{1} \in H_{1}, n \leq n^{*} .
$$

Then, for the solution of (1.9), the a priori estimate

$$
\begin{aligned}
& \left\|u_{1}^{k}\right\|_{H_{1}}^{2}+\frac{1}{\varepsilon}\left\|L_{2,1} u_{1}^{k}\right\|_{H_{2}}^{2} \\
& +\tau \sum_{n=1}^{k}\left(\left\|\bar{\partial}_{0} u_{1}^{n}\right\|_{M_{0}}^{2}+\left\|u_{1}^{n}\right\|_{H_{1}}^{2}+\frac{1}{\varepsilon}\left\|L_{2,1} u_{1}^{n}\right\|_{H_{2}}^{2}\right) \\
& \quad \leq K \tau \sum_{n=1}^{k}\left\|g_{1}^{n}\right\|_{M_{0}}^{2}
\end{aligned}
$$

holds, where the constant $K$ is independent of $k \leq n^{*}$ and $\varepsilon>0$.

Proof By (1.9), we have

$$
\begin{aligned}
& \tau \sum_{n=0}^{k-1}\left(\left(M_{0} \bar{\partial}_{0} u_{1}^{n+1}, \bar{\partial}_{0} u_{1}^{n+1}\right)_{H_{1}}\right. \\
& \left.+\left(L_{1,1} u_{1}^{n+1}, \bar{\partial}_{0} u_{1}^{n+1}\right)_{H_{1}}\right) \\
& +\frac{\tau}{\varepsilon} \sum_{n=0}^{k-1}\left(L_{2,1} u_{1}^{n+1}, L_{2,1} \bar{\partial}_{0} u_{1}^{n+1}\right)_{H_{2}} \\
& \quad=\tau \sum_{n=0}^{k-1}\left(f_{1}^{n+1}, \bar{\partial}_{0} u_{1}^{n+1}\right) H_{1} .
\end{aligned}
$$

The terms in (1.14) involving $L_{1,1}$ and $L_{2,1}$ can be estimated from below in the following manner:

$$
\begin{gathered}
\tau \sum_{n=1}^{k}\left(\left(L_{1,1} u_{1}^{n}, \bar{\partial}_{0} u_{1}^{n}\right)_{H_{1}}+\frac{1}{\varepsilon}\left(L_{2,1} u_{1}^{n}, L_{2,1} \bar{\partial}_{0} u_{1}^{n}\right)_{H_{2}}\right) \\
\geq \frac{\delta}{2}\left\|u_{1}^{k}\right\|_{H_{1}}^{2}+\frac{1}{2 \varepsilon}\left\|L_{2,1} u_{1}^{k}\right\|_{H_{2}}^{2} .
\end{gathered}
$$

The right-hand side of (1.14) is estimated from above as

$$
\begin{aligned}
\tau \sum_{n=1}^{k}\left(f_{1}^{n}, \bar{\partial}_{0} u_{1}^{n}\right)_{H_{1}} & =\tau \sum_{n=1}^{k}\left(g_{1}^{n}, \bar{\partial}_{0} u_{1}^{n}\right)_{M_{0}} \\
& \leq \tau \sum_{n=1}^{k} \frac{1}{2}\left(\left\|\bar{\partial}_{0} u_{1}^{n}\right\|_{M_{0}}^{2}+\left\|g_{1}^{n}\right\|_{M_{0}}^{2}\right)
\end{aligned}
$$

(see (1.12)). Thus, (1.14)-(1.16) lead to

$$
\begin{aligned}
& \frac{1}{2} \tau \sum_{n=1}^{k}\left\|\partial_{0} u_{1}^{n}\right\|_{M_{0}}^{2}+\frac{\delta}{2}\left\|u_{1}^{k}\right\|_{H_{1}}^{2}+\frac{1}{2 \varepsilon}\left\|L_{2,1} u_{1}^{k}\right\|_{H_{2}}^{2} \\
& \quad \leq \tau \sum_{n=1}^{k} \frac{1}{2}\left\|g_{1}^{n}\right\|_{M_{0}}^{2} .
\end{aligned}
$$

By (1.17) and standard inequalities in theory of difference methods (see Ashyralaev and Sobolevskii, 1994; D'yakonov, 1972; Mitchell and Griffiths, 1980; Thomee, 1997), (1.13) follows.

THEOREM 1.4 Let the conditions of Theorem 1.3 be satisfied. Then, for the solution of (1.9), the a priori estimate

$$
\tau \sum_{n=1}^{k}\left\|L_{2,1} u_{1}^{n}\right\|_{H_{2}}^{2} \leq \varepsilon^{2} K \tau \sum_{n=1}^{k}\left(\left\|f_{1}^{n}\right\|_{H_{1}}^{2}+\left\|g_{1}^{n}\right\|_{M_{0}}^{2}\right)
$$

holds, where the constant $K$ is independent of $k \leq n^{*}$ and $\varepsilon>0$.

Proof In accordance with (1.9), we observe that

$$
L_{1,2} L_{2,1} u_{1}^{n}=\varepsilon\left(f_{1}^{n}-M_{0} \bar{\partial}_{0} u_{1}^{n}-L_{1,1} u_{1}^{n}\right) .
$$

By (0.9) and (1.6), we deduce from (1.19)

$$
\begin{aligned}
& \sigma\left\|L_{2,1} u_{1}^{n}\right\|_{H_{2}} \\
& \quad \leq K \varepsilon\left(\left\|f_{1}^{n}\right\|_{H_{1}}+\left\|\bar{\partial}_{0} u_{1}^{n}\right\|_{M_{0}}+\left\|u_{1}^{n}\right\|_{H_{1}}\right) .
\end{aligned}
$$

Therefore, the left-hand side of (1.18) is estimated from above as

$$
K \varepsilon^{2} \tau \sum_{n=1}^{k}\left(\left\|f_{1}^{n}\right\|_{H_{1}}^{2}+\left\|\bar{\partial}_{0} u_{1}^{n}\right\|_{M_{0}}^{2}+\left\|u_{1}^{n}\right\|_{H_{1}}^{2}\right)
$$


(see (1.20)). After that, it suffices to apply the a priori estimates obtained.

Now we indicate a priori estimates for problem (1.5) with $\varepsilon=0$.

THEOREM 1.5 Let the conditions of Theorem 1.1 be satisfied. Then, for the solution of (1.5) with $\varepsilon=0$, the a priori estimates

$$
\begin{aligned}
& \tau \sum_{n=1}^{k}\left\|\bar{\partial}_{0} u_{1}^{n}\right\|_{M_{0}}^{2}+\left\|u_{1}^{k}\right\|_{H_{1}}^{2}+\tau \sum_{n=1}^{k}\left\|u_{1}^{n}\right\|_{H_{1}}^{2} \\
& \quad \leq K \tau \sum_{n=1}^{k}\left\|g_{1}^{n}\right\|_{M_{0}}^{2}, \\
& \tau \sum_{n=1}^{k}\left\|u_{2}^{n}\right\|_{H_{2}}^{2} \leq K \tau \sum_{n=1}^{k}\left(\left\|f_{1}^{n}\right\|_{H_{1}}^{2}+\left\|g_{1}^{n}\right\|_{M_{0}}^{2}\right)
\end{aligned}
$$

hold, where the constant $K$ is independent of $k \leq n^{*}$.

Proof Observe that the second equation in system (1.5) can be rewritten as $L_{2,1} \bar{\partial}_{0} u_{1}^{n+1}=0$. Hence (1.5) implies that

$$
\begin{aligned}
X \equiv & \tau \sum_{n=1}^{k}\left(\left(M_{0} \bar{\partial}_{0} u_{1}^{n}, \bar{\partial}_{0} u_{1}^{n}\right)_{H_{1}}\right. \\
& \left.+\left(L_{1,1} u_{1}^{n}, \bar{\partial}_{0} u_{1}^{n}\right)_{H_{1}}+\varepsilon\left(u_{2}^{n}, u_{2}^{n}\right)_{H_{2}}\right) \\
= & \tau \sum_{n=1}^{k}\left(f_{1}^{n}, \bar{\partial}_{0} u_{1}^{n}\right)_{H_{1}}
\end{aligned}
$$

(see (1.14)). After that the same reasoning as the Proof of Theorem 1.3 leads from (1.23) to (1.21). To prove (1.22), it suffices to rewrite the first equation in system (1.5) as

$$
L_{1,2} u_{2}^{n+1}=f_{1}^{n+1}-M_{0} \bar{\partial}_{0} u_{1}^{n+1}-L_{1,1} u_{1}^{n+1}
$$

and apply evident inequality

$$
\begin{aligned}
\left\|L_{1,2} u_{2}^{n+1}\right\|_{H_{1}} \leq & \left\|f_{1}^{n+1}\right\|_{H_{1}}+\left\|\bar{\partial}_{0} M_{0} u_{1}^{n+1}\right\|_{H_{1}} \\
& +\left\|L_{1,1} u_{1}^{n+1}\right\|_{H_{1}}
\end{aligned}
$$

(see (1.20)) in combination with (0.9) and (1.21).

\subsection{A Priori Estimates and Correctness for More General Problems}

We return now to problem (1.8) in general setting (without the requirement that all $f_{2}^{n}=0$ (see problem (1.5)):

$$
\begin{gathered}
\bar{\partial}_{0} M_{0} u_{1}^{n}+L_{1,1} u_{1}^{n}+L_{1,2} u_{2}^{n}=f_{1}^{n}, \\
L_{2,1} u_{1}^{n}-\varepsilon u_{2}^{n}=f_{2}^{n} .
\end{gathered}
$$

It will also be useful to rewrite it in terms of

$$
\begin{aligned}
& U_{1}^{k} \equiv \tau \sum_{n=0}^{k} u_{1}^{n}, U_{2}^{k} \equiv \tau \sum_{n=0}^{k} u_{2}^{n}, \\
& F_{1}^{k} \equiv \tau \sum_{n=0}^{k} f_{1}^{n}, \quad F_{2}^{k} \equiv \tau \sum_{n=0}^{k} f_{2}^{n}
\end{aligned}
$$

(with $u_{i}^{0}=f_{i}^{0}=0, i=1,2$ ) as

$$
\begin{gathered}
M_{0} u_{1}^{n}+L_{1,1} U_{1}^{n}+L_{1,2} U_{2}^{n}=F_{1}^{n}, \\
L_{2,1} U_{1}^{n}-\varepsilon U_{2}^{n}=F_{2}^{n},
\end{gathered}
$$

$n=1, \ldots, k \leq n^{*} \equiv T / \tau$. It is evident that (1.26) and (1.30) are equivalent. Observe also that $M_{0} u_{1}^{n}=$ $\bar{\partial}_{0} M_{0} U_{1}^{n}, u_{1}^{n}=\bar{\partial}_{0} U_{1}^{n}, u_{2}^{n}=\bar{\partial}_{0} U_{2}^{n}$,

$$
\begin{aligned}
& \left\|U_{i}^{k}\right\|_{H_{i}}^{2} \leq T \tau \sum_{n=0}^{k}\left\|u_{i}^{n}\right\|_{H_{i}}^{2}, \quad i=1,2, \\
& \left\|U_{1}^{k}\right\|_{M_{0}}^{2} \leq T \tau \sum_{n=0}^{k}\left\|u_{i}^{n}\right\|_{M_{0}}^{2} .
\end{aligned}
$$

THEOREM 1.6 Let the conditions of Theorem 1.3 be satisfied. Then, for the solution of (1.25) and (1.26), the a priori estimates

$$
\begin{gathered}
\left\|u_{1}^{k}\right\|_{H_{1}}^{2}+\varepsilon\left\|u_{2}^{k}\right\|_{H_{2}}^{2} \\
+\tau \sum_{n=1}^{k}\left(\left\|\bar{\partial}_{0} u_{1}^{n}\right\|_{M_{0}}^{2}+\left\|u_{1}^{n}\right\|_{H_{1}}^{2}+\varepsilon\left\|u_{2}^{n}\right\|_{H_{2}}^{2}\right) \leq K F_{k} \\
\tau \sum_{n=0}^{k}\left\|u_{2}^{n}\right\|_{H_{2}}^{2} \leq K F_{k}
\end{gathered}
$$


hold, where

$$
F_{k} \equiv \tau \sum_{n=1}^{k}\left(\left\|f_{1}^{n}\right\|_{H_{1}}^{2}+\left\|g_{1}^{n}\right\|_{M_{0}}^{2}\right)+\tau \sum_{n=1}^{k}\left\|\bar{\partial}_{0} f_{2}^{n}\right\|_{H_{2}}^{2}
$$

the constant $K$ is independent of $k \leq n^{*}$ and $\varepsilon \geq 0$.

Proof Restriction (1.26) implies that $L_{2,1} \bar{\partial}_{0} u_{1}^{n}$ $\varepsilon \bar{\partial}_{0} u_{2}^{n}=\bar{\partial}_{0} f_{2}^{n}$. Hence,

$$
\left(L_{2,1} \bar{\partial}_{0} u_{1}^{n}, u_{2}^{n}\right)_{H_{2}}-\varepsilon\left(\bar{\partial}_{0} u_{2}^{n}, u_{2}^{n}\right)_{H_{2}}=\left(\bar{\partial}_{0} f_{2}^{n}, u_{2}^{n}\right)_{H_{2}}
$$

Combining (1.25) and (1.35), we see that

$$
\begin{aligned}
X \equiv \tau & \sum_{n=1}^{k}\left(\left\|\bar{\partial}_{0} u_{1}^{n}\right\|_{M_{0}}^{2}+\left(L_{1,1} u_{1}^{n}, \bar{\partial}_{0} u_{1}^{n}\right)_{H_{1}}\right) \\
& \quad+\varepsilon / 2\left\|u_{2}^{k}\right\|_{H_{2}}^{2} \\
= & \tau \sum_{n=1}^{k}\left(\left(g_{1}^{n}, \bar{\partial}_{0} u_{1}^{n}\right)_{M_{0}}-\left(\bar{\partial}_{0} f_{2}^{n}, u_{2}^{n+1}\right)_{H_{2}}\right) \equiv Y .
\end{aligned}
$$

The left-hand side of (1.36) can be easily estimated as

$$
X \geq \tau \sum_{n=1}^{k}\left\|\bar{\partial}_{0} u_{1}^{n}\right\|_{M_{0}}^{2}+\varepsilon / 2\left\|u_{2}^{k}\right\|_{H_{2}}^{2}+\frac{1}{2} \delta\left\|u_{1}^{k}\right\|_{H_{1}}^{2} .
$$

The first term on the right-hand side of (1.36) presents no problems since we can make use of inequality

$$
\left(g_{1}^{n}, \bar{\partial}_{0} u_{1}^{n}\right)_{M_{0}} \leq \frac{1}{4}\left\|\bar{\partial}_{0} u_{1}^{n}\right\|_{M_{0}}^{2}+\left\|g_{1}^{n}\right\|_{M_{0}}^{2}
$$

The second term on the right-hand side of (1.36) is

$$
\begin{gathered}
Z \equiv-\tau \sum_{n=1}^{k}\left(\bar{\partial}_{0} f_{2}^{n}, u_{2}^{n}\right)_{H_{2}} ; \\
|Z| \leq \tau \sum_{n=1}^{k}\left\|\bar{\partial}_{0} f_{2}^{n}\right\|_{H_{2}}\left\|u_{2}^{n}\right\|_{H_{2}},
\end{gathered}
$$

where $\left\|u_{2}^{n}\right\|_{H_{2}}$ is estimated from above via (1.24) and (0.9). Thus,

$$
\begin{aligned}
|Z| \leq & \kappa \tau \sum_{n=1}^{k}\left(\frac{\alpha}{2}\left\|\bar{\partial}_{0} u_{1}^{n}\right\|_{M_{0}}^{2}+\left(1+\frac{2}{\alpha}\right)\left\|\bar{\partial}_{0} f_{2}^{n}\right\|_{H_{2}}^{2}\right. \\
& \left.+\left\|f_{1}^{n}\right\|_{H_{1}}^{2}+\left\|u_{1}^{n}\right\|_{H_{1}}^{2}\right)
\end{aligned}
$$

with a $\kappa>0$ and small enough $\alpha>0$. Combination of (1.38) and (1.39) yields the desired estimate for $Y$ in (1.36) and a basic consequence of (1.36) and (1.37), which leads to (1.32) and (1.34). Inequality (1.33) follows from (1.25) and (1.32) (see the Proof of (1.22)).

THEOREM 1.7 Let the conditions of Theorem 1.3 be satisfied. Then, for the solution of (1.29) and (1.26), the a priori estimates

$$
\begin{gathered}
\tau \sum_{n=1}^{k}\left\|u_{1}^{n}\right\|_{M_{0}}^{2}+\left\|U_{1}^{k}\right\|_{H_{1}}^{2}+\varepsilon\left\|U_{2}^{k}\right\|_{H_{2}}^{2} \leq K \bar{F}_{k} \\
\tau \sum_{n=0}^{k}\left\|U_{2}^{n}\right\|_{H_{2}}^{2} \leq K \bar{F}_{k}
\end{gathered}
$$

hold, where

$$
\bar{F}_{k} \equiv \tau \sum_{n=1}^{k}\left(\left\|f_{1}^{n}\right\|_{H_{1}}^{2}+\left\|g_{1}^{n}\right\|_{M_{0}}^{2}\right)+\tau \sum_{n=1}^{k}\left\|f_{2}^{n}\right\|_{H_{2}}^{2}
$$

the constant $K$ is independent of $k \leq n^{*}$ and $\varepsilon \geq 0$.

Proof This is just a repeat of the Proof of Theorem 1.6, with obvious modifications connected with the use of $(1.27)-(1.31)$ to obtain (1.40)-(1.42) from (1.32)-(1.34).

Note that all results obtained can be easily formulated for other Hilbert spaces $H_{1}$ and $H_{2}$. What is of importance is that $M_{0} \in \mathcal{L}\left(H_{1} ; H_{1}\right), \quad M=$ $M^{*}>0$ and that Theorem 1.1 applies; hence elasticity and hydrodynamics problems with $L_{2,1} \equiv \operatorname{div}$ can be mentioned as examples (see D'yakonov, 1996a and references therein). We note also that the case $M_{0} \asymp I_{1}$ is allowed. Instead of $I_{2}$, we can 
deal with

$$
L_{2,2} \in \mathcal{L}\left(H_{2} ; H_{2}\right), \quad L_{2,2}=L_{2,2}^{*} \geq 0
$$

then $\varepsilon\left\|u_{2}\right\|_{H_{2}}^{2}$ in our estimates should be replaced by $\varepsilon\left\|u_{2}\right\|_{L_{2,2}}^{2} \equiv \varepsilon\left(L_{2,2} u_{2}, u_{2}\right)_{H_{2}}$. For example, the choice

$$
\begin{gathered}
H_{1} \equiv G_{1,1}\left(\Omega ; \Gamma ; \Gamma_{0}\right) ; \quad H_{2} \equiv H^{1}\left(\Gamma ; \Gamma_{0}\right) \\
\left\|u_{2}\right\|_{L_{2,2}}^{2} \equiv\left|u_{2}\right|_{0, \Gamma_{0}}^{2}
\end{gathered}
$$

is of a special importance when we wish to preserve the homogeneous Dirichlet conditions on $\Gamma_{0} \subset \Gamma$; here $\Gamma_{0}=\bar{\Gamma}_{0}$ is a union of several arcs; $H^{1}\left(\Gamma ; \Gamma_{0}\right)$ is a subspace of $H^{1}(\Gamma)$ whose elements vanish on $\Gamma_{0}$ and $G_{1,1}\left(\Omega ; \Gamma ; \Gamma_{0}\right)$ corresponds to a subspace of $G_{1,1}(\Omega ; \Gamma)$ with elements having traces in $H_{2}$ (see D'yakonov, 1997b). Note finally that more general problems with nonsymmetric operators are possible (see $(0.12))$.

\subsection{Nonstationary Boundary Conditions}

Instead of the conditions $L_{2,1} u_{1}^{n}-\varepsilon L_{2,2} u_{2}^{n}=f_{2}^{n}$ in (1.5) and (1.26), new conditions,

$$
L_{2,1} \bar{\partial}_{0} u_{1}^{n}-\varepsilon \bar{\partial}_{0} L_{2,2} u_{2}^{n}=f_{2}^{n}
$$

are considered here (see (1.43) and (1.44)) with $u_{2}^{0}=$ 0 for simplicity of exposition. (The original parabolic problems in strengthened Sobolev spaces with nonstationary boundary conditions of type (1.45) might be of special importance in competition with the homogeneous Dirichlet conditions on $\Gamma_{0}$.)

THEOREM 1.8 Let the conditions of Theorem 1.3 be satisfied. Then, for the solution of (1.25) and (1.45), the a priori estimates

$$
\begin{gathered}
\left\|u_{1}^{k}\right\|_{H_{1}}^{2}+\varepsilon\left\|u_{2}^{k}\right\|_{L_{2,2}}^{2} \\
+\tau \sum_{n=1}^{k}\left(\left\|\bar{\partial}_{0} u_{1}^{n}\right\|_{M_{0}}^{2}+\left\|u_{1}^{n}\right\|_{H_{1}}^{2}+\varepsilon\left\|u_{2}^{n}\right\|_{L_{2,2}}^{2}\right) \leq K F_{k} \\
\tau \sum_{n=0}^{k}\left\|u_{2}^{n}\right\|_{H_{2}}^{2} \leq K F_{k}
\end{gathered}
$$

hold, where

$$
F_{k} \equiv \tau \sum_{n=1}^{k}\left(\left\|f_{1}^{n}\right\|_{H_{1}}^{2}+\left\|g_{1}^{n}\right\|_{M_{0}}^{2}+\left\|f_{2}^{n}\right\|_{H_{2}}^{2}\right)
$$

the constant $K$ is independent of $k \leq n^{*}$ and $\varepsilon \geq 0$.

Proof It is easy to see that the corresponding analog of Theorem 1.1 holds. Thus the solution of (1.25) and (1.45) exists and is unique. To prove (1.46) and (1.47) we apply the Proof of Theorem 1.6. Observe that (1.35) should be replaced by

$$
\left(L_{2,1} \bar{\partial}_{0} u_{1}^{n}, u_{2}^{n}\right)_{H_{2}}-\varepsilon\left(\bar{\partial}_{0} u_{2}^{n}, u_{2}^{n}\right)_{L_{2,2}}=\left(f_{2}^{n}, u_{2}^{n}\right)_{H_{2}}
$$

no $\bar{\partial}_{0} f_{2}^{n}$ is needed here. This enables us to apply the Proof of Theorem 1.6 with slight alterations.

Note also that the case of conditions

$$
L_{2,1} \bar{\partial}_{0} u_{1}^{n}-\varepsilon L_{2,2} u_{2}^{n}=f_{2}^{n}
$$

is analogous to the considered one.

THEOREM 1.9 Let the conditions of Theorem 1.3 be satisfied. Then, for the solution of (1.25) and (1.48), the a priori estimate

$$
\begin{aligned}
& \tau \sum_{n=1}^{k}\left\|\bar{\partial}_{0} u_{1}^{n}\right\|_{M_{0}}^{2}+\left\|u_{1}^{k}\right\|_{H_{1}}^{2}+\tau \sum_{n=1}^{k}\left\|u_{1}^{n}\right\|_{H_{1}}^{2} \\
& \quad+\tau \sum_{n=0}^{k}\left\|u_{2}^{n}\right\|_{H_{2}}^{2} \leq K F_{k}
\end{aligned}
$$

holds, where

$$
F_{k} \equiv \tau \sum_{n=1}^{k}\left(\left\|f_{1}^{n}\right\|_{H_{1}}^{2}+\left\|g_{1}^{n}\right\|_{M_{0}}^{2}+\left\|f_{2}^{n}\right\|_{H_{2}}^{2}\right)
$$

the constant $K$ is independent of $k \leq n^{*}$ and $\varepsilon \geq 0$.

Proof This is just a repeat of the Proofs of Theorem 1.8 and 1.6, with obvious modifications connected with the use of

$$
\left(L_{2,1} \bar{\partial}_{0} u_{1}^{n}, u_{2}^{n}\right)_{H_{2}}-\varepsilon\left(u_{2}^{n}, u_{2}^{n}\right)_{L_{2,2}}=\left(f_{2}^{n}, u_{2}^{n}\right)_{H_{2}}
$$

instead of (1.35). 


\section{PERTURBATION THEOREMS}

\subsection{Perturbation Theorems for Parabolic Problems}

Here, we study dependence of the solution of type (1.9) and (1.8) (or (1.29) and (1.26)) on the parameter $\varepsilon$ when $\varepsilon \rightarrow+0$. We denote by $z_{i}^{n}, i=1,2$ the difference $u_{\varepsilon, i}^{n}-u_{0, i}^{n}$, where index $\varepsilon$ is now used to indicate the corresponding problem and its solution. We also make use of

$$
Z_{1}^{k} \equiv \tau \sum_{n=0}^{k} z_{1}^{n}, \quad Z_{2}^{k} \equiv \tau \sum_{n=0}^{k} z_{2}^{n}
$$

THEOREM 2.1 Let the conditions of Theorem 1.7 be satisfied and suppose that all $F_{k} \leq K^{*}$ with $K^{*}$ independent of $k \leq n^{*}$. Then, for the solution of (1.25) and (1.26), we have

$$
\tau \sum_{n=1}^{k}\left\|z_{1}^{n}\right\|_{M_{0}}^{2}+\left\|Z_{1}^{k}\right\|_{H_{1}}^{2}+\tau \sum_{n=0}^{k}\left\|Z_{2}^{n}\right\|_{H_{2}}^{2} \leq K \varepsilon^{2},
$$

where the constant $K$ is independent of $k \leq n^{*}$ and $\varepsilon \geq 0$.

Proof In accordance with (1.25) and (1.26), we have

$$
\begin{gathered}
\bar{\partial}_{0} M_{0} z_{1}^{n}+L_{1,1} z_{1}^{n}+L_{1,2} z_{2}^{n}=0, \\
L_{2,1} z_{1}^{n}=\varepsilon u_{\varepsilon, 2}^{n} \equiv f_{2}^{n} .
\end{gathered}
$$

These relations (2.3) and (2.4), together with (2.1), imply that

$$
\begin{gathered}
\bar{\partial}_{0} M_{0} Z_{1}^{n}+L_{1,1} Z_{1}^{n}+L_{1,2} Z_{2}^{n}=0, \\
L_{2,1} Z_{1}^{n}=\varepsilon \tau \sum_{n=0}^{k} u_{\varepsilon, 2}^{n} \equiv F_{2}^{n} .
\end{gathered}
$$

Hence, Theorem 1.7 for (2.5) and (2.6) leads directly to (2.2).

It is worth noting that Theorem 2.1 implies asymptotically optimal $(O(\varepsilon))$ convergence in the norm the square of which is given by the lefthand term of (2.2); the use of Theorem 1.6 and the norm defined by (1.32) and (1.33) leads only to
$O(\varepsilon / \tau)$-convergence. There is also a possibility to establish $O\left(\varepsilon^{1 / 2}\right)$-convergence for problem (1.9).

THEOREM 2.2 Let the conditions of Theorem 1.3 be satisfied and suppose that

$$
\tau \sum_{n=1}^{k}\left(\left\|f_{1}^{n}\right\|_{H_{1}}^{2}+\left\|g_{1}^{n}\right\|_{M_{0}}^{2}\right) \leq K^{*}
$$

with $K^{*}$ independent of $k \leq n^{*}$. Then, for the solution of (1.9), we have

$$
\left\|z_{1}^{k}\right\|_{M_{0}}^{2}+\tau \sum_{n=1}^{k}\left\|z_{1}^{n}\right\|_{H_{1}}^{2} \leq K \varepsilon
$$

where the constant $K$ is independent of $k \leq n^{*}$ and $\varepsilon>0$.

Proof By (1.9) and (1.5) with $\varepsilon=0$, we have

$$
\bar{\partial}_{0} M_{0} z_{1}^{n}+L_{1,1} z_{1}^{n}+\frac{1}{\varepsilon} L_{1,2} L_{2,1} z_{1}^{n}=L_{1,2} u_{0,2}^{n} .
$$

Therefore,

$$
\begin{aligned}
& \tau \sum_{n=1}^{k}\left(\left(\partial_{0} z_{1}^{n}, z_{1}^{n}\right)_{M_{0}}+\left(L_{1,1} z_{1}^{n}, z_{1}^{n}\right)_{H_{1}}+\frac{1}{\varepsilon}\left\|L_{2,1} z_{1}^{n}\right\|_{H_{2}}^{2}\right) \\
& \quad=\tau \sum_{n=1}^{k}\left(u_{0,2}^{n}, L_{2,1} z_{1}^{n}\right)_{H_{1}} .
\end{aligned}
$$

Since $\left(u_{0,2}^{n}, L_{2,1} z_{1}^{n}\right)_{H_{1}} \leq \varepsilon^{-1}\left\|L_{2,1} z_{1}^{n}\right\|_{H_{2}}^{2}+4 \varepsilon\left\|z_{1}^{n}\right\|_{H_{1}}^{2}$, we can conclude from (2.9) and (2.7) that (2.8) holds.

Analogously, perturbation theorems can be obtained on the basis of Theorem 1.8 (1.9) for parabolic problems in strengthened Sobolev spaces with nonstationary boundary conditions.

\subsection{Splitting of the Region for Parabolic Problems}

We start by considering a partition of $\bar{\Omega}$ into a set of blocks (panels) $\bar{\Omega}_{1}, \ldots, \bar{\Omega}_{i^{*}}$; each domain $\Omega_{i}$ has a Lipschitz piecewise smooth boundary $\Gamma_{i} \equiv \partial \Omega_{i}$ 
and the Sobolev space $H^{1}\left(\Gamma_{i}\right)$ can be easily defined (all its elements are equivalent to continuous functions).

On the set $S \equiv \bigcup_{i} \partial \Omega_{i}$ (the union of the panel boundaries) we define the Hilbert space $H_{2} \equiv H_{2}(S)$ with the square of the norm

$$
\|g\|_{H_{2}}^{2} \equiv \sum_{i=1}^{i^{*}}\|g\|_{1, \Gamma_{i}}^{2}
$$

$\mathrm{H}_{2}$ consists of functions $\mathrm{g} \in L_{2}(S)$ such that they are equivalent to continuous functions on $S$ and their restrictions to each $\Gamma_{i}$ belong to $H_{1}\left(\Gamma_{i}\right)$.

The model strengthened Sobolev space

$$
G_{1,1} \equiv G_{1,1}(\Omega ; S) \equiv H_{1}
$$

consists of functions in $H^{1}(\Omega)$ such that their traces on each $\Gamma_{i}$ belong to $H^{1}\left(\Gamma_{i}\right)$, so we may define

$$
\|v\|_{H_{1}}^{2} \equiv\|v\|_{H^{1}(\Omega)}^{2}+\sum_{i=1}^{i^{*}}\left\|\operatorname{Tr}_{\Gamma_{i}} v\right\|_{H^{1}\left(\Gamma_{i}\right)}^{2} .
$$

It is known that $H_{1}$ is a Hilbert space and the trace operator $\operatorname{Tr}_{S} \in \mathcal{L}\left(H_{1} ; L_{2}(S)\right)$ can be considered as an element of $\mathcal{L}\left(H_{1} ; H_{2}(S)\right)$; moreover, $\operatorname{Tr}_{S} \in$ $\mathcal{L}\left(H_{1} ; H_{2}(S)\right)$ is normally invertible operator (see D'yakonov, 1996a; 1997b). This key fact enables us to apply results indicated above to problems in the strengthened Sobolev space $H_{1}$ (see (2.11) and (2.12)) and in the Hilbert space $H \equiv H_{1} \times H_{2}$ (see (2.10)). For example, Theorem 1.9 applies for (1.25) with conditions (on $S$ )

$$
L_{2,1} \bar{\partial}_{0} u_{1}^{n}-\varepsilon u_{2}^{n}=0
$$

the case $\varepsilon=0$ corresponds to split (with respect to the original partition of $\bar{\Omega}$ ) problems dealing with each panel separately (with the homogeneous Dirichlet conditions on each $\Gamma_{i}$ ); the corresponding perturbation estimate is

$$
\tau \sum_{n=1}^{k}\left\|\bar{\partial}_{0} z_{1}^{n}\right\|_{M_{0}}^{2}+\left\|z_{1}^{k}\right\|_{H_{1}}^{2}+\tau \sum_{n=0}^{k}\left\|u_{2}^{n}\right\|_{H_{2}}^{2} \leq K \varepsilon^{2}
$$

Finally, we note that hyperbolic problems deserve a separate study, but it is fairly clear that modifications of the results indicated above are possible.

\section{References}

Ashyralaev, A. and Sobolevskii, P.E. (1994). Wellposedness of Parabolic Difference Equations. Birkhauser, Basel, p. 346.

Babushka, I. (1973). The finite element method with penalty. Math. Comput. 27, 221-228.

Babushka, I. (1987). The finite element method with Lagrangian multipliers. Numer. Math. 20, 179-193.

Bramble, J. (1981). The Lagrange multiplier method for Dirichlet's problem. Math. Comp. 37, 1-11.

Brezzi, F. (1974). On the existence, uniqueness and approximation of saddle-point problems arising from Lagrangian multipliers. Rev. Fran. Automat. Inf. Recherche Operationelle. Ser. Rouge. Anal. Numer. 2, 129-151.

Ciarlet, P. (1997). Mathematical Elasticity. Volume II: Theory of Plates. Studies in Mathematics and Its Applications, 27, NH, Amsterdam, p. 497.

Cosserat, Eugene et Francois. (1898). Sur les equations de la theorie de l'elastite. C. R. Acad. Sci. (Paris) 126, 1089-1091.

Courant, R. (1943). Variational methods for the solution of problems of equilibrium and vibrations. Bull. Amer. Math. Soc. 49, 1-23.

Duong, T.H. and Joly, P. (1994). On the stability analysis of boundary conditions for the wave equation by energy methods. Math. Comp. 62, 539-563.

D'yakonov, E.G. (1972). Difference Methods for Solving Boundary Value Problems. 2 (Nonstationary Problems). Moscow State University, Moscow, p. 227 (in Russian).

D'yakonov, E.G. (1973). On Stability of difference schemes for some nonstationary problems. In: Topics in Numerical Analysis, Vol. II (J.J.H. Miller, Ed.), Academic Press, London, pp. $63-87$.

D'yakonov, E.G. (1983). Estimates of computational work for boundary value problems with the Stokes operators. Soviet math. (Iz. VUZ) 27, 57-71.

D'yakonov, E.G. (1986). Effective methods for solving eigenvalue problems with fourth-order elliptic operators. Soviet $J$. Numer. Anal. Math. Modelling 1, 59-82.

D'yakonov, E.G. (1996a). Optimization in Solving Elliptic Problems. CRC Press, Boca Raton, p. 590.

D'yakonov, E.G. (1996b). Effective numerical methods for solving elliptic problems in strengthened Sobolev spaces. Seventh Copper Mountain Conference on Multigrid Methods, 1996. NASA-CP. 3339, pp. 199-213.

D'yakonov, E.G. (1997a). A new approach to the Dirichlet boundary conditions based on using strengthened Sobolev spaces. Doklady Mathematics 55, 106-109.

D'yakonov, E.G. (1997b). On the Dirichlet boundary conditions in spectral problems for projective-grid elliptic operators. Vestnik Moskov. Univ., Ser. Vychisl. Mat. i Kibernet. N 4, 17-24 (in Russian).

D'yakonov, E.G. (1997c). Asymptotically optimal algorithms for problems of the Stokes type in strengthened Sobolev spaces. Doklady Mathematics 56, 571-573. 
D'yakonov, E.G. (1997d). Operator problems in strengthened Sobolev spaces and numerical methods for them. Lecture Notes in Computer Science Vol. 1196, pp. 161-169.

Girault, V. and Raviart, P.A. (1986). Finite Elements Methods for Navier-Stokes Equations. Theory and Algorithms. Springer, Berlin, p. 371

Glowinski, R. (1983). Numerical Methods for Nonlinear Variational Problems. Springer-Verlag, New York, p. 493.

Kobelkov, G.M. (1994). On numerical methods of solving the Navier-Stokes equations in velocity-pressure variables. In: Numerical Methods and Applications (G.I. Marchuk, Ed.), CRC Press, Boca Raton, pp. 81-115.

Krein, S.G. (1971). Linear Equations in Banach Spaces. Nauka, Moscow, p. 104 (in Russian).

Mitchell, A.R. and Griffiths, D.F. (1980). The Finite Difference Methods in Partial Differential Equations. John Wiley, Chichester, p. 272.

Pironneeau, O. (1989). Finite Element Methods for Fluids. John Wiley, Chichester, p. 212.
Rempel, S. and Schulze, B.W. (1985). Index Theory of Elliptic Boundary Problems. North Oxford Academic, Oxford, p. 393.

Sheen, D. (1993). Second-order absorbing boundary conditions for the wave equation in a rectangular domain. Math. Comp. 61, 599-606.

Sobolevskii, P.E. (1981). An approximation of the Stokes equations by Neumann's problems. Chisl. Metody Mekh. Sploshnoi Sredy 12, 124-139 (in Russian).

Sobolevskii, P.E. and Vasil'ev, V.V. (1978). On an $\epsilon$-approximation of the Navier-Stokes equations. Chisl. Metody Mekh. Sploshnoi Sredy 9, 115-139 (in Russian).

Thomee, V. (1997). Galerkin Finite Element Methods for Parabolic Problems. Springer-Verlag, Berlin, p. 302.

Trenogin, V.A. (1980). Functional Analysis. Nauka, Moscow, p. 495 (in Russian).

Vishik, M.I. (1970). Sobolev-Slobodeckii spaces of variable order with weighted norms and their application to elliptic mixed boundary value problems. In: Partial Differential Equations. Nauka, Moscow, pp. 71-76 (in Russian). 


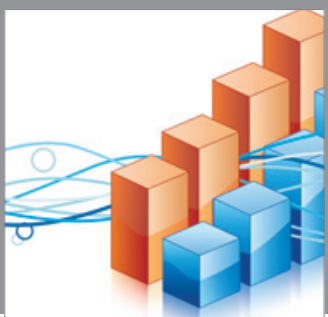

Advances in

Operations Research

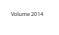

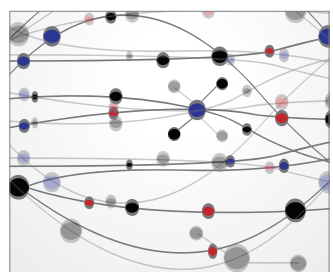

\section{The Scientific} World Journal
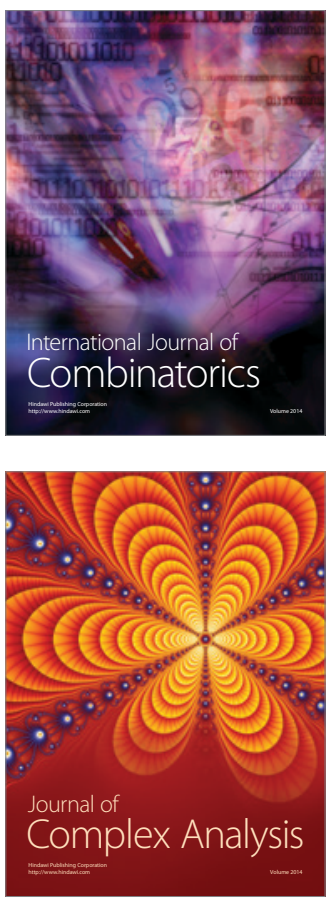

International Journal of

Mathematics and

Mathematical

Sciences
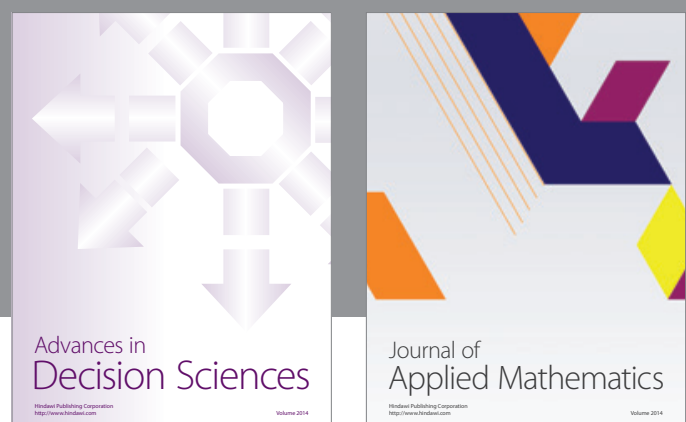

Journal of

Applied Mathematics
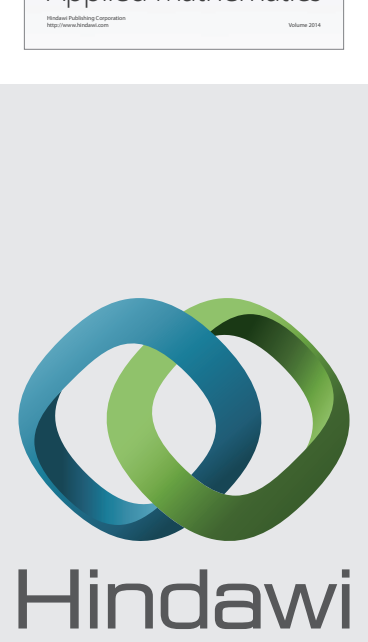

Submit your manuscripts at http://www.hindawi.com
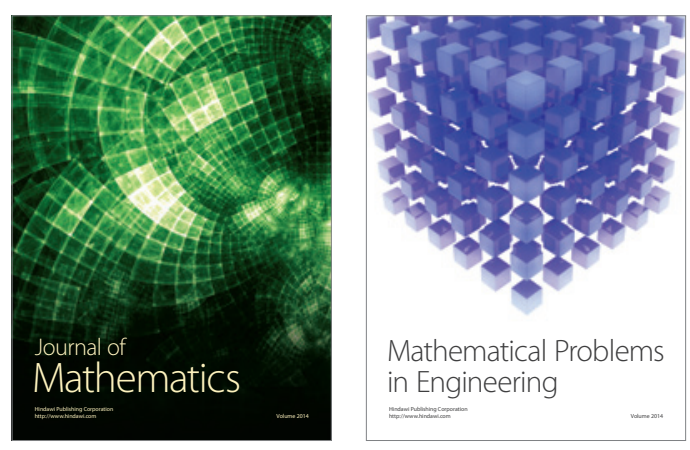

Mathematical Problems in Engineering
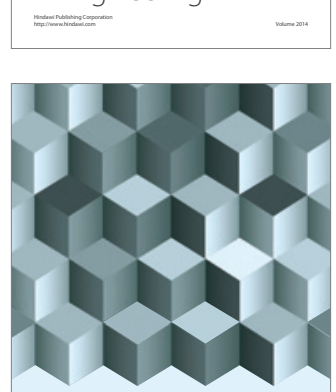

Journal of

Function Spaces
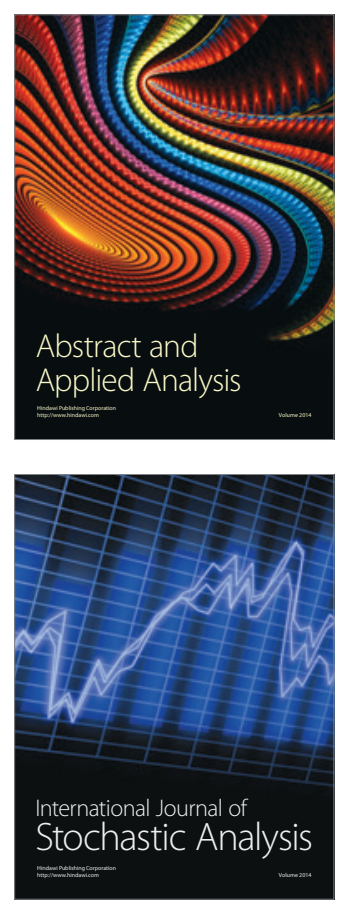

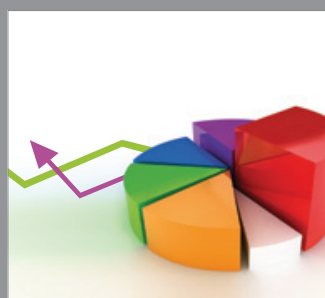

ournal of

Probability and Statistics

Promensencen
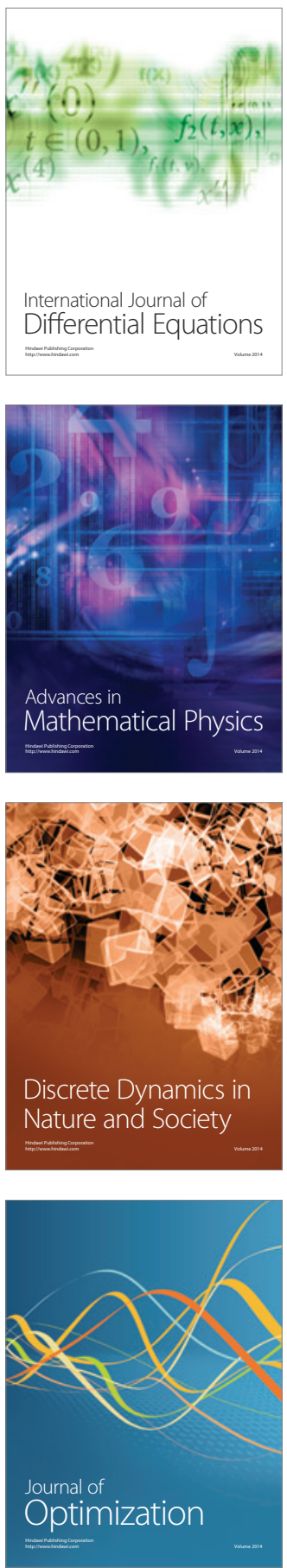